Décadrages Décadrages

cınéma, à travers champs Cinéma, à travers champs

$14 \mid 2009$

Cinéma et migration

\title{
Entre esthétisme et militantisme : la figure du saisonnier dans le « nouveau » cinéma suisse
}

Marthe Porret

\section{(2) OpenEdition}

1 Journals

Édition électronique

URL : https://journals.openedition.org/decadrages/325

DOI : $10.4000 /$ decadrages.325

ISSN : 2297-5977

Éditeur

Association Décadrages

Édition imprimée

Date de publication : 1 avril 2009

Pagination : 28-38

ISBN : 978-2-9700582-9-8

ISSN : 2235-7823

Référence électronique

Marthe Porret, «Entre esthétisme et militantisme : la figure du saisonnier dans le « nouveau » cinéma suisse », Décadrages [En ligne], 14 | 2009, mis en ligne le 10 avril 2010, consulté le 03 avril 2022. URL : http://journals.openedition.org/decadrages/325 ; DOI : https://doi.org/10.4000/decadrages.325 


\title{
Entre esthétisme et militantisme: la figure du
}

\author{
saisonnier dans le "nouveau " cinéma suisse
}

par Marthe Porret

1 Cf. Norbert Ledergerber, Urs Jaeggi, Solothurner Filmtage 1966-1985: Geschichte und Entwicklung, Universitätsverlag Freiburg, 1985. Le débat principal voit ainsi s'affronter Niklaus Gessner, qui vient montrer son film Un milliard dans un billard (1964) et qui ne croit qu'à l'avenir d'un cinéma commercial, divertissant et avec vedettes, à Alexander J. Seiler, qui a présenté de son côté Siamo Italiani (1964) et qui défend un cinéma d'auteurs.

2 Alexander J. Seiler signe en 1978 un article précisément intitulé: "Die entfremdete Heimat. Entwurf einer Perspektive zum neuen Schweizer Film" ["La patrie aliénée. Ebauche de perspective du nouveau cinéma suisse"], in Film in der Schweiz, Carl Hanser Verlag, München/Wien, pp. 7-42, où il revient notamment sur l'histoire de la réalisation de Siamo Italiani.
L'histoire économique de la Suisse depuis la fin de la Seconde Guerre mondiale jusqu'à aujourd'hui est intimement liée à celle de son cinéma. Non pas que la période de haute conjoncture générale ne signifiât la mise en place d'une industrie cinématographique florissante, bien au contraire. On sait que les tout débuts d'une aide publique au cinéma national ne se mettent en place qu'à partir de janvier 1963, et que celleci ne concerne que les films dits "culturels", ou documentaires. On sait également que la fin des années 1960 voit les trois grandes maisons de production existantes encore actives dans la fiction de long métrage (Praesens, Gloria Film et Condor Film) perdre du terrain ou disparaître complètement. Pour les nouvelles structures de production qui apparaissent dans les années 1960 et qui se lancent dans le long métrage indépendant, le marché intérieur (petitesse et plurilinguisme) ne leur permet pas d'y exister durablement. Le cinéma suisse «d'auteurs» - en opposition à la production de commande - est un cinéma subventionné. Dans le cas contraire, il doit s'expatrier, donc "s'internationaliser»: c'est en ces termes qu'un débat vif s'empart des Premières Journées de Soleure en janvier 19661. Le boom économique ne profite donc pas à la production cinématographique nationale qui reste un secteur économique d'une importance négligeable. Les cinéastes, s'ils veulent travailler régulièrement, doivent s'expatrier. Quant à ceux qui restent et qui choisissent de faire un cinéma moins cher - et par là même techniquement plus léger - il faut souligner qu'ils souhaitent pour la majorité d'entre eux redécouvrir les réalités de leur patrie ${ }^{2}$. En effet, l'idée selon laquelle l'ancien cinéma suisse n'avait donné du pays que des images factices et sans lien avec les problèmes contemporains est récurrente au sein de l'intelligentsia helvétique: romanciers, dramaturges, d'abord, puis journalistes, critiques de cinéma et cinéastes appellent de leurs vœux un "nouveau cinéma suisse» en prise avec la réalité du pays. 
Non, si le cinéma n'est que très peu soutenu par l'économie du pays, en revanche, l'histoire économique de la Suisse se reflète largement dans son cinéma. Le propos de cet article est donc d'appréhender le film en tant que vecteur d'images sociales ${ }^{3}$, en se concentrant plus spécifiquement sur la figure du travailleur immigré dans les années 1960 et 1970, ou sur ce que l'on a longtemps désigné plus précisément par le terme de "saisonnier». Après la Seconde Guerre mondiale, la Suisse comme tous ses pays voisins, connaît en effet un fort développement qui se traduit notamment par une hausse de la consommation et de la croissance démographique. On modernise et on construit donc à tour de bras routes, barrages hydrauliques et immeubles d'habitation. Pour pallier au manque de main d'œuvre, le gouvernement suisse délivre des permis de travail, dits "Permis A», donnant aux immigrés la permission de travailler durant neuf mois par an en Suisse, avec l'obligation de retourner chez eux pendant trois mois ${ }^{4}$. Leur afflux de plus en plus important dès la fin des années 1950 est perçu par certains comme un facteur de perturbation de l'équilibre social. Les saisonniers sont ainsi érigés en problème pour une population suisse qui vit alors en pleine euphorie consumériste. Le dépôt en 1969 de la célèbre Initiative Schwarzenbach 5 puis les dérives xénophobes qu'elle entraîne poussent les cinéastes à prendre parti et expliquent que nombre d'entre eux vont dénoncer à leur façon - qui n'est pas celle de la génération actuelle $\mathbf{6}$ - les conditions objectivement déplorables des ouvriers venus travailler ici. Il est à cet égard très intéressant de constater avec Seiler que le thème dominant de ce cinéma des années 1960 et 1970 est celui d'une dialectique récurrente entre patrie et étranger ${ }^{7}$. Ce que ne manque pas non plus de remarquer le critique Martin Schaub, en se penchant sur le contenu récurrent des longs métrages suisses du "nouveau cinéma suisse»: parmi les neuf thématiques retenues, la figure de l'étranger y trouve bonne place 8 .

C'est donc sur la figure du saisonnier que nous allons nous pencher pour voir comment son traitement formel et politique a évolué. Une analyse fine des représentations sociopolitiques du saisonnier au sein $\mathrm{du}$ "nouveau» cinéma suisse implique de travailler sur des films représentatifs du sujet en utilisant une méthode qui tienne compte des spécificités du langage filmique. C'est pourquoi nous allons être attentifs non seulement aux choix narratifs et aux points de vue adoptés, mais il s'agira aussi de prendre en compte la mise en scène des "acteurs» et des «décors», le type de cadrage ainsi que le traitement de la bande son, en mettant l'accent sur les divers procédés de montage. Le corpus comprend trois films suisses : Siamo Italiani - die Italiener (1964) d'Alexander J. Seiler, Rob Gnant et June Kovach, Le Train rouge (1973) de Peter
3 Laurent Guido adopte une démarche similaire dans l'étude des représentions de la peur dans le cinéma américain (Les peurs de Hollywood, Antipodes, Lausanne, 2006, p. 27).

4 Le statut interdit par ailleurs de changer d'employeur, de louer son propre logement et de faire venir conjoint et enfants. Entre la fin des années 1940 et juin 2002, date à laquelle ce statut de saisonnier a été aboli grâce à l'accord avec I'Union européenne sur la libre circulation des personnes, près de 7 millions de permis $\mathrm{A}$ seront délivrés.

5 L'initiative présidée par James Schwarzenbach est déposée le 20 mai 1969 auprès du gouvernement. Son but est de réduire de 10\% dans chaque canton (à Genève de 25\%) le nombre d'étrangers en Suisse en quatre ans. Le Conseil fédéral, puis l'Assemblée fédérale la rejettent. Avec 71772 signatures, elle doit néanmoins être soumise à votation populaire. Le 7 juin 1970, elle est refusée par 654588 Non contre 557714 Oui.

6 Voir l'article de Séverine Graff dans ce même numéro.

$\mathbf{7}$ "Die Dialektik von Heimat und entfremdung, in der ich das Grundmotiv des neuen Schweizer films sehe, [...].", Alexander J. Seiler, op. cit., p. 8.

8 L'usage de la liberté. Le nouveau cinéma suisse 1964-1984, L'Age d'Homme/Pro Helvetia, Lausanne, 1985, chapitre 5: "Etranger, mon miroir", pp. 73-82. Les autres thèmes sont: l'évocation critique du passé immédiat de la Suisse, l'aspiration à un ordre économique et social disparu, le désir de partir et l'obligation de rester, la figure du marginal, l'image de la femme, le documentaire comme conscience sociale de la nation, le goût formel de ce cinéma pour la mesure et sa pudeur de contenu, et enfin les types de cadres et lieux d'action choisis. 
9 Freddy Buache, Le cinéma suisse, L'Age d'Homme, Lausanne, 1974, [1998]; Film in der Schweiz (Bernhard Giger et al.), op. cit.; Werner Wider et Felix Aeppli, Der Schweizer Film 19291964, Band 1 und 2, Limmat Verlag, Zurich, 1981; Martin Schaub, L'usage de la liberté: le nouveau cinéma suisse 1964-1984, op. cit.; et Martin Schlappner, Martin Schaub, Cinéma Suisse. Regards critiques 1896-1987, Centre Suisse du Cinéma, 1987.

10 Nous avons travaillé à partir du transfert DVD des deux copies $16 \mathrm{~mm}$ existantes déposées à la Cinémathèque suisse.

11 Pour ce qui est de leurs carrières publiques, Siamo Italiani ne trouve pas de distributeur; en revanche il est sélectionné - et récompensé aux festivals documentaires de Bilbao et Florence en 1965 puis projeté à Soleure l'année suivante. Le Train rouge est montré en 1973 aux festivals de Soleure et de Locarno (mention spéciale du jury des jeunes). Les Cahiers de la Cinémathèque, $\mathrm{n}^{\circ} 12$, hiver 1974, pp. 8691 , rendent compte du débat passionné à l'issue de sa projection à la Chaux-de-Fonds dans le cadre du $13^{\mathrm{e}} \mathrm{CICl}$. II sort ensuite à Berne, Genève, Renens, Zurich et Paris. Buseto a été projeté aux festivals de Venise en 1974, Soleure, Locarno et Figueira da Foz en 1975 avant de connaître une petite exploitation à Zurich et Berne. Cf. Histoire du cinéma suisse 1966-2000, Hervé Dumont, Maria Tortajada (éd.), Cinémathèque suisse/Gilles Attinger, Lausanne/Hauterive, 2007, t. I, pp. 9-11, 152153 et $159-160$.

12 Ammann (Braccia si, uomini no, 1970; Les Neinsager, 1971; Pourquoi la drogue?, 1980) comme Legnazzi (Chronik von Prugiasco, 1978; Zweiter Anfang, 1981; My Mother Is in Sri Lanka, 1987) se consacrent tous deux à des problématiques sociales telles que l'immigration, l'exode rural ou les toxicomanes en Suisse.

13 Histoire du cinéma suisse 1966-2000, op. cit., p. 11.

14 ld., p. 10.

15 Gazette littéraire, 17-18.10.64.
Ammann et Buseto - die Emigration am Beispiel eines sizilianischen Dorfes (1974) de Remo Legnazzi. Le premier est d'accès facile car disponible maintenant en DVD (Filmcoopi Zürich, 2006). Mais il est surtout considéré dans la majorité des publications relatives au "nouveau» cinéma suisse comme le film qui, avec Les Apprentis (1964) d'Alain Tanner et les films d'Henry Brandt pour l'Exposition nationale de 1964, marque précisément les débuts historiques de ce nouveau cinéma ${ }^{9}$. Il est aussi à notre connaissance le premier long métrage de cinéma consacré au sujet. Pour évaluer l'impact des mouvements d'extrême gauche et de la radicalisation des débats sur les films sur l'immigration qui ont suivi, nous avons retenu Le Train rouge et Buseto ${ }^{10}$. En effet, même s'ils n'ont pas eu une carrière critique de même ampleur que le film de Seiler11, ils sont, d'une part, réalisés peu après la votation populaire sur la surpopulation étrangère - ou initiative Schwarzenbach - de juin 1970 par des réalisateurs qui, d'autre part, privilégient un cinéma documentaire en lien avec l'actualité socio-politique de leur pays $\mathbf{1 2}$.

\section{Siamo Italiani et le "cinéma-vérité"}

Après quelques courts métrages publicitaires, Seiler, Gnant et Kovach signent avec Siamo Italiani leur premier long métrage. Le sujet, en prise avec la réalité helvétique contemporaine, n’a encore jamais été traité au cinéma. Ses audaces formelles frappent aussi les esprits. A ce titre, la critique suisse en fait une étape historique dans l'histoire du cinéma suisse13. Sa dimension politique réside dans le fait qu'il se démarque clairement de l'ancien cinéma suisse, notamment par la volonté, nouvelle à l'époque, de faire du cinéma un outil d'analyse de la société. Pour autant, aux dires de ses trois réalisateurs, Siamo Italiani ne se veut pas partisan: «[notre film] n'est d'aucune tendance et ne représente aucun parti. Il ne s'occupe pas du 〈problème〉 socio-économique, mais des êtres humains en tant qu'individus vivants et concrets.»14 Par ailleurs, dans leurs déclarations, il faut souligner le fait qu'ils se revendiquent du "cinéma-vérité» tel qu'il se développe alors en France, au Canada et aux Etats-Unis. Il est vrai que leur démarche est identique à Richard Leacock ou Robert Drew : $16 \mathrm{~mm}$, peu d'éclairage artificiel, entretiens, instance de filmage qui cherche à se faire oublier, immersion dans le milieu, absence de musique d'accompagnement. Mais c'est aussi pour s'en démarquer :

"Notre technique diffère du cinéma-vérité en ce sens que nous n'avions pas mené d'enquête préalable. Nous sommes partis sans idées préconçues; nous n'avons pas voulu isoler des 〈héros〉, mais faire en sorte que les travailleurs racontent eux-mêmes l'histoire anonyme des Italiens en Suisse. »15 


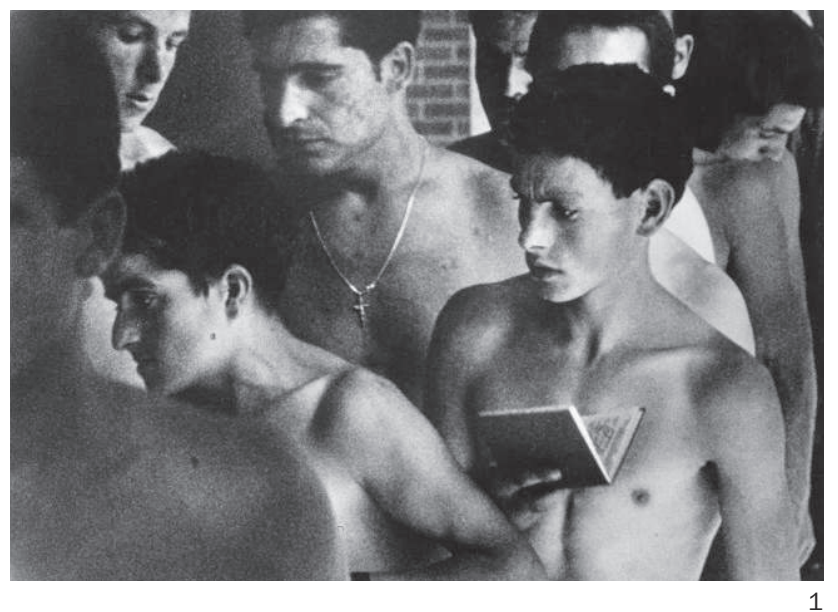

De fait, c'est effectivement, d'une manière générale dans ce film, une accumulation dans l'image de visages inconnus (fig. 1), ou alors la succession, pour ce qui est de la bande son, de témoignages anonymes. Ainsi, au sein de la séquence dédiée à la visite sanitaire, deux pistes sonores sont mixées ensemble: à l'appel continuel, dans un micro, des hommes par leur nom et prénom se superposent un instant des extraits tirés des entretiens ("on m’a dit que c'était bien en Suisse, alors...»). Il s'agit donc de "racont[er], sans acteurs et sans premier rôle, l'histoire banale et anonyme du travailleur italien en Suisse»16. Mais témoigner du quotidien rapproche aussi Seiler, Kovach et Gnant des jeunes cinéastes contemporains actifs alors dans le domaine de la fiction : "Il ne s'agit plus de raconter une histoire selon le mode classique, mais de saisir la banalité de la vie de tous les jours. "17 De fait - et de façon assez inattendue - de nombreuses séquences évoquent immédiatement l'univers 16 Histoire du cinéma suisse 1966-2000, op. cit., p. 11.

17 Ibid. des films de Cassavetes (Shadows, 1959), surtout lorsqu'il s'agit de filmer les loisirs et les sorties nocturnes du week-end. Ainsi la très longue séquence tournée dans une boîte de striptease a le pouvoir de nous abstraire temporairement du reste du film tant la prise sur le vif des images révèle un microcosme humain passionnant (circonscrit entre les W.C. où se préparent les danseuses, le vestiaire et la salle). De leur côté, les images de foire et notamment d'auto-tamponneuses sont celles que l'on pourrait trouver dans certains films de la Nouvelle Vague comme Les 400 coups de Truffaut (1959). Et de manière générale, typiquement, les seules musiques que l'on entend sont celles, diégétiques, de la foire, du bal ou du cabaret (reprises yéyé, jazz,...). 

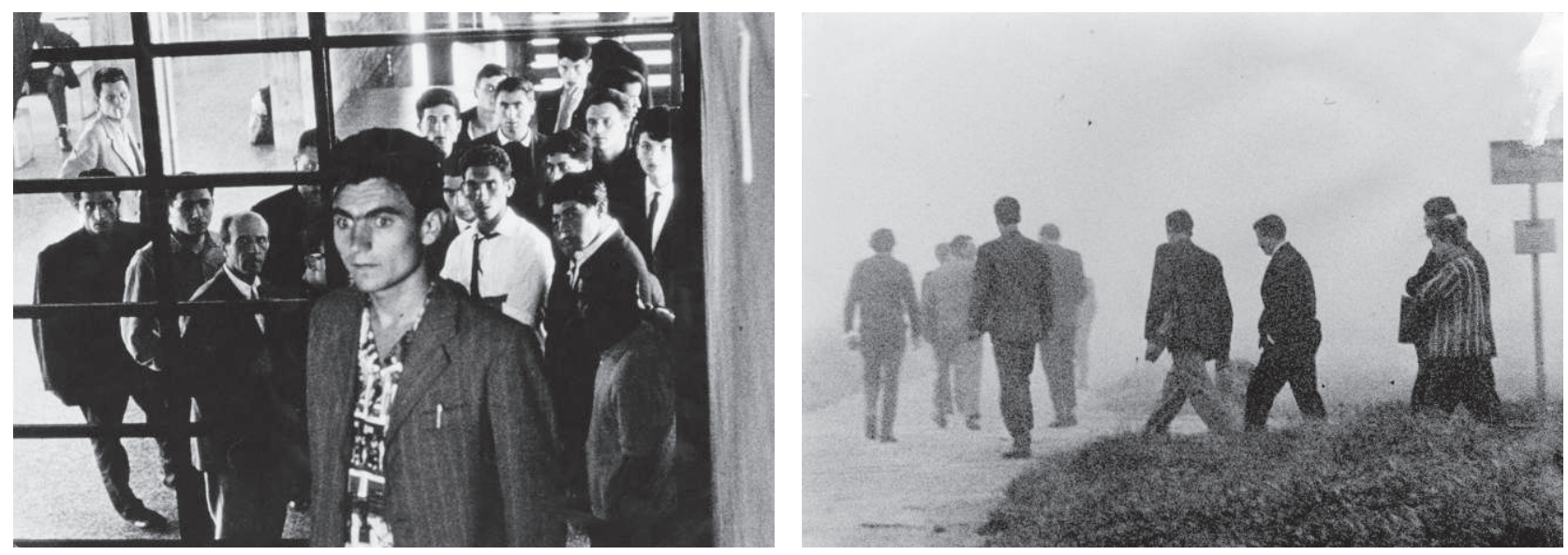

2

Par ailleurs, la grande qualité plastique des images noir blanc au grain visible (conséquence du gonflage en $35 \mathrm{~mm}$ ) fait pencher le film encore davantage du côté d'un cinéma d'abstraction. Les gros plans sur les visages, les nombreux contre-jours et les plans filmant les silhouettes sur un fond clair constituent des références aux premières avant-gardes française et russe (fig. 2 et 3). La première séquence en usine, au moment où des hommes tractent de longues pièces d'acier, est, nous semble-t-il, explicite: de façon inattendue, répétitions et chevauchements du plan font alors patiner l'action pour mieux en dénoncer la dimension aliénante. La séquence du pré-générique, qui juxtaposent les gros plans de saisonniers qui se présentent ("Je m'appelle Carla,...", - chaque plan étant coupé systématiquement après le prénom - joue là aussi, de façon évidente, avec ce plaisir esthétique de la répétition visuelle et sonore. Mais Siamo Italiani, qui traite d'un sujet d'actualité, recourt aussi aux formes plus classiques, donc didactiques, du documentaire. Certes, avec parcimonie. Ainsi, un carton initial pose brièvement mais efficacement les termes de l'assimilation - ou plutôt de la non assimilation - de la

18 "Plus de 500000 Italiens vivent et travaillent en Suisse. Ils passent pour un , problème . Une économie en haute-conjoncture a besoin de leur main-d'œuvre - un petit peuple aux particularités prononcées les tient isolés derrière la barrière d'une autre langue. En tant que sproblème, ils sont l'objet de discussions - en tant qu'être humains ils restent des inconnus. " (carton d'ouverture). main-d'œuvre étrangère en Suisse $\mathbf{1 8}$, tandis qu'une voix over masculine donne, ici ou là, des explications utiles.

Ce premier film de cinéma consacré au sujet qui nous intéresse frappe surtout par le soin accordé à sa beauté formelle. Sa caractéristique principale, en termes de discours sur les immigrés, est certes de montrer concrètement comment ils vivent, ou tuent le temps, au quotidien. Mais le traitement esthétisant qui en est fait prouve que les réalisateurs ne cherchent pas tant à dénoncer qu'à jouer de cette fascination pour l'étranger. Le saisonnier, mis à distance par une série de procédés fil- 
miques, reste un Etranger. Les rapports très travaillés entre bandes son et image mettent précisément en évidence l'impossible intégration des saisonniers dans notre société. Un exemple parmi d'autres: le film donne la parole - et non l'image - aux habitants suisses en déclinant les uns après les autres les lieux communs qui circulent sur les Italiens: "huit Suisses font moins de bruit que deux Italiens!», "On a l'impression que c'est nous, les étrangers!", "Ils consomment beaucoup trop!", "Ils se jettent sur les femmes suisses!»...). A l'image apparaissent des ouvriers, désœuvrés, qui fument en groupe en observant le flux des passants pressés (fig. 4). La fracture entre saisonniers et citoyens helvétiques, qui est mise en avant par cette déliaison entre bandes son et images, cherche moins à dénoncer cette scission qu'à isoler l'étranger, représenté dans une perspective quasi "exotisante».

\section{Dix ans plus tard...}

En l'espace de dix ans, on assiste en Suisse comme partout dans le monde à une radicalisation des mouvements d'extrême gauche. La question de la surpopulation étrangère polarise les débats aussi bien dans les partis en place qu'au sein des mouvements militants. Les citoyens suisses, appelés à se prononcer sur le sujet en 1970 puis en 1974, sont par ailleurs régulièrement informés des conditions de travail des saisonniers via les reportages d'actualités télévisées qui se succèdent de façon impressionnante 19. Peter Ammann et René Burri signent notamment pour la télévision Braccia si, uomini no (1970), un reportage qui couvre la campagne précédant la votation du 7 juin 1970, la célèbre "initiative Schwarzenbach». Suisses et étrangers de tous bords politiques s'y
19 Voir à ce sujet le film d'Alex Mayenfisch, Statut: saisonnier (DVD Climage, TSR, 2003), qui fait un travail de montage à partir des archives de la TSR de 1960 à 1990.

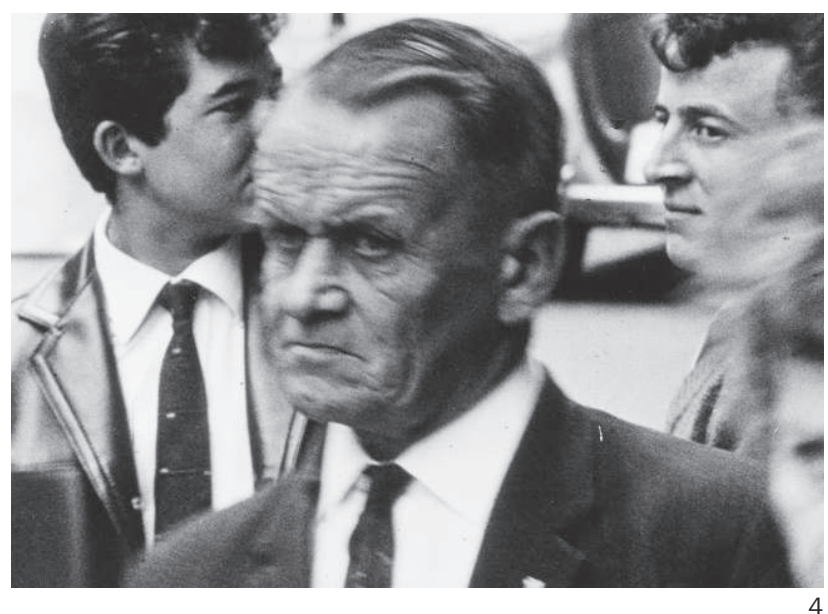




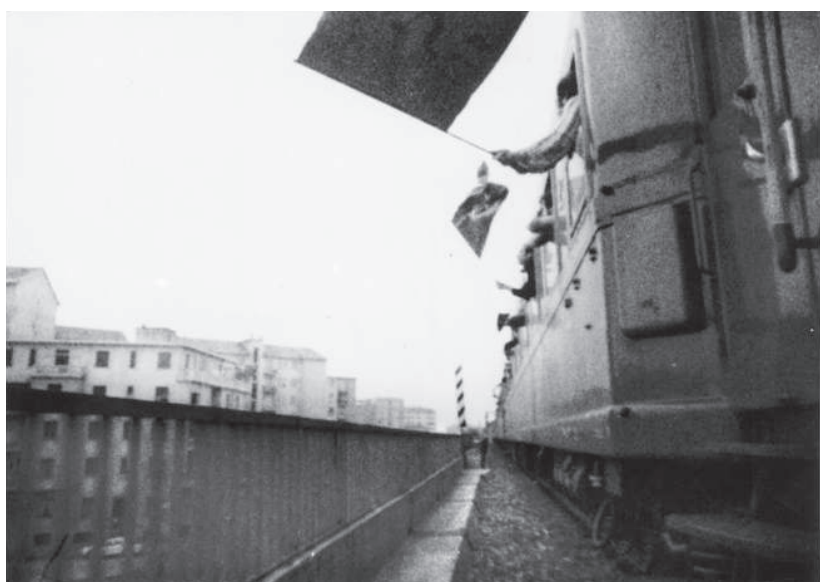

5

expriment publiquement. En 1973, Ammann continue à traiter cette thématique avec son deuxième long métrage, Le Train rouge. Legnazzi réalise Buseto l'année suivante. Ces films, on va le voir, participent aussi de cette polarisation et médiatisation du débat, mais procèdent différemment pour ce qui est de convaincre le spectateur suisse.

La structure fondamentale du Train rouge fonctionne sur le principe de l'alternance, et cela à plusieurs niveaux. Le fil rouge (au sens quasi littéral!) est constitué par un des trains spéciaux au départ de Suisse qui rallie le Sud de l'Italie, avec à son bord les travailleurs émigrés qui rentrent au pays pour pouvoir voter (fig. 5). La caméra, très mobile, suit en particulier - nous offrant parfois, par dessus son épaule, son point de vue - un militant communiste dont le mot d'ordre est: "Votez contre ceux qui vous obligent à émigrer!». On joue ici, contrairement à Siamo Italiani, sur une possible identification avec l'Etranger. A partir de là, plusieurs séquences hétérogènes se déploient et s'entrecroisent en parallèle, induisant chez le spectateur une lecture métaphorique. En effet, quatre d'entre elles sont directement liées au mythe fondateur de la Confédération helvétique: on assiste à la représentation en plein air à Interlaken du drame de Friedrich Schiller Wilhelm Tell, à celle du Guglielmo Tell de Rossini à l'opéra de Florence, à la pièce moderne d'Alfonso Sastre Gli occhi tristi di Guglielmo Tell interprétée par une troupe d'amateurs sardes, et enfin à la visite d'une classe d'écoliers suisses allemands sur les lieux historiques de la plaine du Grütli. Ammann monte ensuite en alternance toutes les séries, tissant entre elles des liens concrets. Le parallèle le plus évident pour tout spectateur suisse (et explicitement formulé par les ouvriers italiens dans le film) est 
le suivant: l'Italie des années 1970, comme la Suisse du XIII ${ }^{\mathrm{e}}$ siècle, subit encore le joug de ses rois et barons (grands propriétaires fonciers, Vatican et grand patronat); le serment d'alliance entre Confédérés rebelles est un exemple à suivre pour ce pays. Mais l'intelligence du montage va dialectiser ce propos en montrant dans un premier temps que les souffrances (factices!) endurées par les paysans suisses dans le drame de Schiller sont dérisoires à côté de la dureté du travail bien réel des ouvriers italiens qui percent un tunnel ou travaillent sur un chantier. A la représentation théâtrale - un peu empesée et maladroite - d'un accident de travail qui va pousser nos ancêtres suisses à se rebeller succèdent par exemple les images d'une grue qui s'est effondrée sur un chantier et la levée d'un cercueil. Mais le jeu du montage va même plus loin puisqu'il montre que la Suisse elle-même n'a plus rien à voir avec l'imagerie folklorique, mythologisante ou romantique véhiculée par l'art: une visite au Musée suisse de l'horlogerie au Locle est suivie par les images du travail à la chaîne qui est devenue l'industrie horlogère suisse.

Le deuxième type d'alternance, et c'est là une caractéristique fondamentale par rapport à un film antérieur comme Siamo Italiani, réside dans le fait que le pays d'origine des immigrés apparaît maintenant à l'image, en alternance avec la Suisse. Une série de séquences tournées en Italie du Sud pendant la campagne précédant les élections permet d'en montrer plusieurs aspects tout en venant encore enrichir le jeu des parallélismes et des métaphores: une procession catholique qui draine une foule impressionnante - et à laquelle participe d'ailleurs un des saisonniers en tant que pénitent - fait écho aux différentes représentations de Guillaume Tell, mais se confond aussi avec le cortège de manifestants communistes, renvoyant dos à dos mythe, religion et politique.

Le «pluriculturalisme» dont fait preuve Ammann et qui se traduit par un montage alternant se retrouve aussi dans Buseto ${ }^{20}$. De même que le discours public sur l'immigration ne se pense alors plus en termes d'assimilation ni en termes d'opposition du national au non national, ces deux cinéastes, filmant ici et là-bas (fig. 6), font exister simultanément deux réalités sociales différentes. Mais là où Ammann articule dialectiquement avec brio une multitude de séquences hétérogènes entre elles, Legnazzi, qui utilise uniquement deux séries séquentielles (ici : Buseto, un village de Sicile, là-bas: la Suisse), signe un documentaire classique de facture didactique, alourdissant littéralement son propos en doublant d'une voix over de longs cartons explicatifs, le tout entrant souvent en redondance avec l'image elle-même. Mais ces «défauts » vont de pair avec cette nouvelle volonté d'aller regarder au-delà de la frontière suisse pour comprendre et reconstruire le fil de cette migration. Il s'agit
20 "En l'occurrence, et pour simplifier beaucoup, il s'agit du passage d'une perception des réalités sociales en termes d'assimilation à une perception en termes de pluriculturalisme/ multiculturalisme. Aujourd'hui, le terme d'assimilation est, par définition, largement critiqué, démonétisé, rejeté, voire moqué parce qu'il serait totalement dépassé, nostalgique, réactionnaire, etc.", Uli Windisch, Suisseimmigrés: quarante ans de débats 1960-2001, L'Age d'Homme, Lausanne, 2002, p. 184. Cette notion de pluriculturalisme ne date pas des années 1970, mais on voit bien qu'Ammann va déjà dans ce sens. 


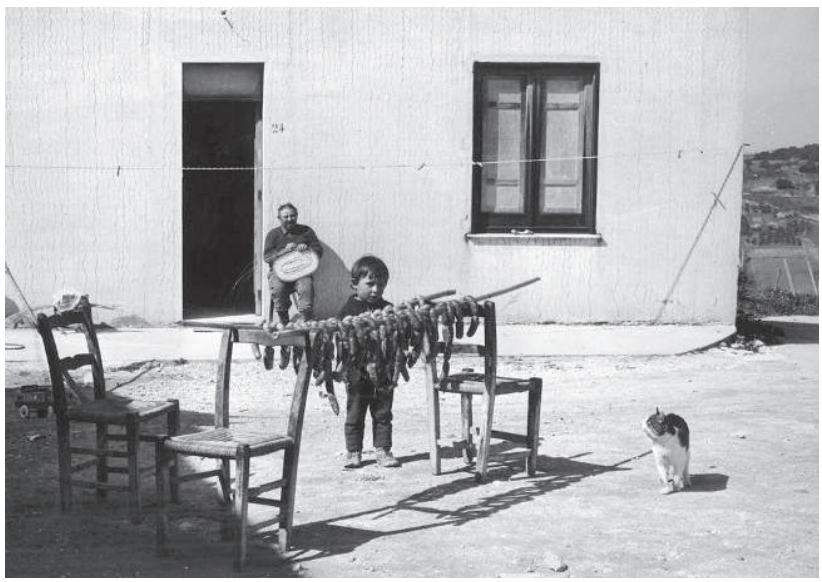

6

d'expliquer au spectateur suisse les tenants et aboutissants du phénomène d'une part, et de relier à une réalité humaine ce qui n'est en Suisse qu'une main-d'œuvre anonyme d'autre part. On cherche, comme dans Le train rouge, à ce que le spectateur puisse se rapprocher et s'identifier aux personnes filmées.

Le premier but se traduit donc, on l'a dit, par une utilisation extensive des cartons explicatifs ("Des 4500 habitants, 1200 ont émigré, dont 800 en Suisse.»), des plans descriptifs (travail agricole sans machine) et du commentaire informatif ( La moitié des habitants de Buseto habite et travaille sur une terre qui appartient aux grandes famille de propriétaires fonciers.»). Legnazzi donne, au travers de trois portraits individualisés, les raisons de l'émigration sicilienne (fig. 7). Mais en choisissant comme point de départ Buseto, il montre les conséquences que cette émigration entraîne sur le village lui-même. En termes économiques tout d'abord: des 21 cordonniers que comptait par exemple le village en 1960, il n'en reste plus qu'un. En revanche, les émigrés ont donné du travail aux artisans locaux en faisant construire de nouvelles maisons au pays. En termes humains, ensuite, les portraits de famille permettent dans un deuxième temps de rendre compte de la façon concrète dont femme et enfants vivent au quotidien la séparation d'avec le père de famille. Et c'est là que la figure du montage alterné - qui fait exister deux espaces filmiques différents en simultané - se montre la plus à même de traduire visuellement l'absurde isolement que vivent en parallèle mari et femme. Une séquence en particulier joue d'avantage que le reste du film sur cette idée de simultanéité : des images du mari écrivant à sa femme en dehors des heures de travail sont relayées par 

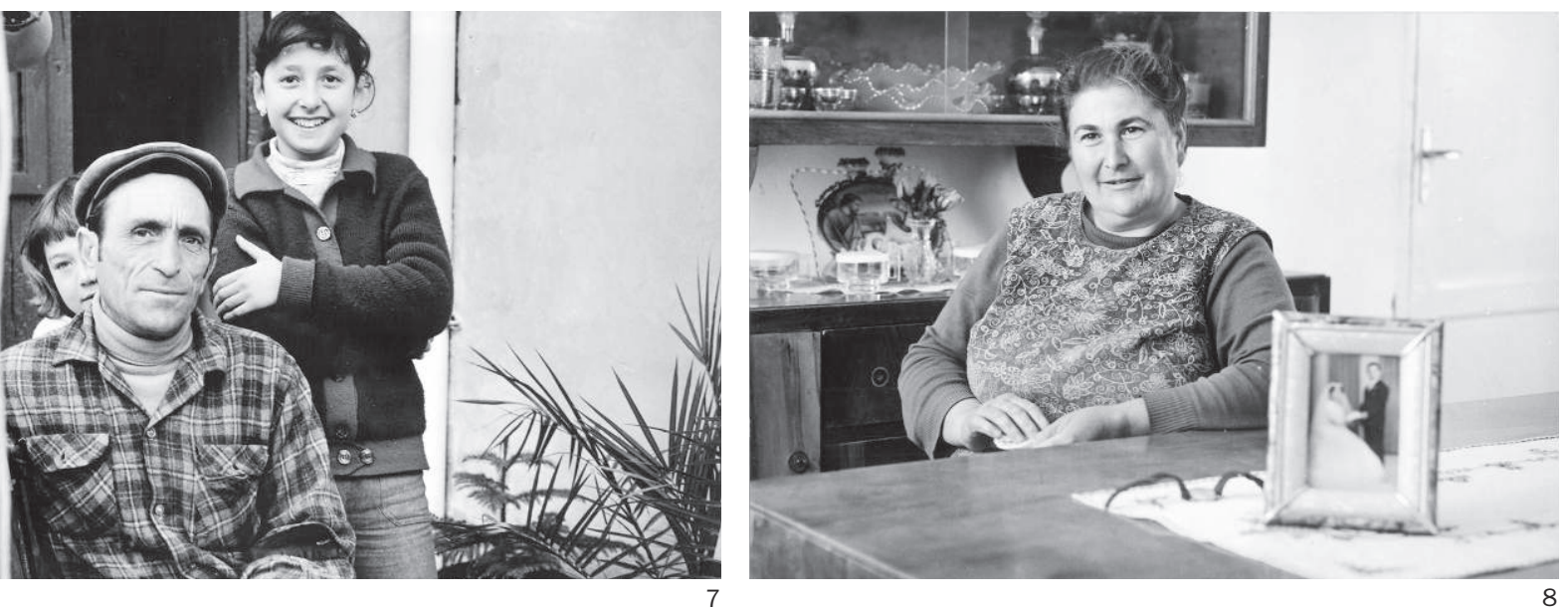

celles de sa femme lisant cette même lettre dans l'espace de sa cuisine (fig. 8).

Contrairement au Train rouge qui exploite davantage le registre émotionnel, Buseto, on le voit, tente de convaincre le spectateur au moyen d'arguments purement rationnels. Cette volonté de démonstration vient peut-être prendre le contre-pied de l'initiative Schwarzenbach dont le contenu, objectivement difficilement réalisable, exploitait largement le domaine de l'émotionnel.

\section{Fascination, émotion ou démonstration}

Siamo Italiani, Le Train rouge et Buseto permettent, grâce au recul des années, de voir à quel point le contexte culturel (pratiques de tournage, style visuel en vogue) et politique (radicalisation progressive du débat) marque les œuvres. Formellement, on l'a vu, cela se traduit par une pratique documentaire marquée dans un premier temps par les innovations d'un cinéma plus «direct». Le traitement esthétique qui est fait du saisonnier - et qui joue au niveau narratif sur le contraste entre étrangers et autochtones - montre à quel point la figure de l'étranger vivant en Suisse fascine tout d'abord dans ce qu'elle manifeste de non national. Aucun phénomène d'identification n'est alors possible. Puis les techniques de cinéma léger s'imposent largement via la télévision publique suisse qui va à son tour régulièrement relayer le débat politique auprès des citoyens. Deux directions différentes se dessinent alors : un cinéma militant, comme celui d'Amman, qui tout en reprenant à son compte le point de vue des immigrés veut faire avancer leur cause; et un cinéma didactique, celui de Legnazzi, certes plus fastidieux et aujourd'hui passé 
de mode, mais qui responsabilise le spectateur en tâchant de lui donner toutes les cartes en mains pour qu'il se forge sa propre opinion. Mais dans les trois cas, nous semble-t-il, une prise de position politique est assumée par le cinéaste dès lors que celui-ci décide de ne donner la parole - et l'image - qu'au saisonnier. Car derrière le saisonnier, c'est la figure plus générale de l'ouvrier qui se cache. Ou plutôt qui s'exprime, devrait-on dire, et le plus souvent, dans ces films, avec une conscience aiguë des injustices que sa condition de classe entraîne. Il n'est ainsi pas étonnant de voir au générique de Buseto que le film se présente sous l'égide de la Centrale suisse d'éducation ouvrière (die Schweizerische Arbeiterbildungszentrale); ou qu'Alexander Seiler signe, après Siamo Italiani, le documentaire Die Früchte der Arbeit (1977), retraçant l'histoire du mouvement ouvrier suisse de 1914 à 1974. Dans les années 1960 et 1970, la figure du migrant au cinéma ne se comprend, consciemment ou non, qu'en termes de lutte des classes. 